\title{
GESTÃO DE PESSOAS NA PERSPECTIVA DE GESTORES DAS COORDENADORIAS REGIONAIS DO MUNICÍPIO DE SÃO PAULO
}

\section{PEOPLE MANAGEMENT ON THE PERSPECTIVE OF MANAGERS FROM THE REGIONAL COORDENATIONS OF THE CITY OF SÃO PAULO}

\author{
Alessandra Paula Ferreira Moreira Neumann ${ }^{1 *}$, Mariana Cabral Schveitzer ${ }^{2}$, Virginia Junqueira ${ }^{3}$ \\ ${ }_{1}$ Doutora em Ciências, Pós-doutoranda da Universidade Federal de São Paulo, São Paulo, SP, Brasil, ale11.neumann@gmail.com \\ 2Pós-doutorado em Enfermagem, Docente da Universidade Federal de São Paulo, São Paulo, SP, Brasil, marycabral101@gmail.com \\ ${ }^{3}$ Pós-doutorado em Economia Política da Saúde, Docente da Universidade Federal de São Paulo, Baixada Santista, SP, Brasil, \\ virginiaj@uol.com.br \\ *Autora de correspondência
}

\section{Resumo}

Mudanças significativas ocorreram na administração pública brasileira, particularmente a contar da década de 1990 , alegadamente em busca de maior efetividade e eficiência na reorganização do aparelho estatal. A administração pública deve contar com áreas competentes na gestão de pessoas, objetivando promover políticas e ações que garantam a qualidade do ambiente do trabalho. Essa pesquisa objetivou compreender como os gestores públicos das coordenadorias regionais da Secretaria Municipal de Saúde de São Paulo vivenciam a gestão de pessoas no que concerne aos serviços de saúde pública. Trata-se de uma pesquisa descritiva, qualitativa, realizada no município de São Paulo, no período de 2015 à 2017. Participaram da pesquisa seis gestores, por meio de entrevistas semiestruturadas que foram submetidas à análise de conteúdo. Percebeu-se que havia descontentamento na forma de condução da gestão de pessoas, especialmente no que se referia às contratações, e existiam demandas de maior divulgação e transparência. Verificaram-se esforços conjuntos dos gestores para melhoria nas relações de trabalho e maior cobrança no que dizia respeito aos critérios de ocupações de cargos.

Palavras-chave: Políticas de pessoal. Gestão. Coordenadorias. Transparência.

\begin{abstract}
Significant changes have occurred in Brazilian public administration, particularly since the 1990s, supposedly in search of greater effectiveness and efficiency in the reorganization of the state apparatus. The public administration must have competent areas in social management, aiming to promote policies and actions that guarantee the quality of the work environment. This research aimed to understand how public managers of the regional coordinators of the Municipal Health Secretariat of São Paulo experience social management regarding public health services. This is a descriptive, qualitative research carried out in the city of São Paulo from 2015 to 2017. Six managers participated in the survey, through semi-structured interviews that were submitted to content analysis. It was noticed that there was discontent in the way of conducting social management, especially with regard to hiring, and there were demands for greater disclosure and transparency. There were joint efforts by managers to improve working relationships and greater demands in terms of job occupation criteria.
\end{abstract}

Keywords: Personnel policies. Management. Coordinators. Transparency. 


\section{INTRODUÇÃO}

Nas últimas décadas, a administração pública no Brasil tem mantido um discurso de busca de maior efetividade e eficiência, alegadamente em busca da reorganização do aparelho estatal. Foram adotados modelos que impactaram a gestão de pessoas, com redução das funções anteriormente cumpridas pelo Estado e transferência da gestão para setores privados, sem que, no entanto, tenha ocorrido organização e fortalecimento da capacidade de regulação pelo poder público.

No entanto, vários autores assinalam que não ocorreu organização e fortalecimento da capacidade de regulação pelo poder público Tendo como ponto de partida o 'modelo burocrático', mudanças significativas ocorreram na administração pública brasileira particularmente a contar da década de 1990, culminando na ideia da eficiência como princípio regente da lógica gerencialista neoliberal voltada para resultados, ocorrendo progressivamente, na sequência, a colonização do setor público (BRESSER-PEREIRA, 1998; COHN, 2009; FERREIRA e ARAUJO, 2014).

As mudanças mundiais de ordem econômica, política, social e cultural, sob a égide do neoliberalismo, impõem formas de conceber e praticar a gestão. Embora as leis, decretos, normas visem formalmente o bem comum, tal dimensão de ordem macropolítica não contempla a determinação social que envolve as relações de trabalho, privilegiando a avaliação da qualidade do serviço prestado sob um enfoque quantitativo de metas e produção (LACAZ e JUNQUEIRA, 2017).

Atualmente os profissionais que atuam na área de gestão de pessoas em saúde defrontamse com uma prática de grande complexidade, diante do desafio da busca da universalidade e a equidade na prestação de serviços; bem como a necessidade de intermediar os processos decisórios (SCHRAIBER, 1999).

Surgem as críticas relativas aos modelos vigentes, incluindo os relativos à Gestão de Recursos Humanos na área da saúde, e o termo Gestão de pessoas foi incorporado na década de 1990, passando o profissional a ser chamado de colaborador da Instituição, devendo ser comprometido e responsável. Objetiva-se o reconhecimento do trabalhador como agente transformador de seu ambiente e não apenas um realizador de tarefas (MACHADO e FILHO, 2014).

Sendo assim, entende-se que as políticas de gestão na área da saúde representam também ações e procedimentos de interesse público. Cada setor da administração deve buscar um fortalecimento institucional incluindo espaços para discussão, negociação e pactuação entre gestores do sistema, usuários e trabalhadores (BRASIL, 2002).

A partir desse cenário, surgiu a indagação de como os gestores das coordenadorias regionais da Secretaria Municipal de Saúde de São Paulo percebiam essa temática, e, assim, objetiva-se compreender como estes agentes públicos vivenciam a gestão de pessoas no setor da saúde pública.

\section{ASPECTOS DAS COORDENADORIAS REGIONAIS DE SAÚDE}

As prefeituras têm um papel importante nos procedimentos internos de contratação e concursos públicos, bem como a na distribuição dos trabalhadores públicos nos devidos lugares, gerenciando as folhas de pagamento, férias e exonerações de servidores públicos. Podem também atuar na gestão da publicação do diário oficial, licitações e compras (SÃO PAULO, 2012).

Os servidores públicos do Município de São Paulo contam com um Estatuto, a lei no 8.989, de 29 de outubro de 1979, considerada a maior lei do funcionalismo público municipal, com direitos, 
deveres, regras de assuntos voltados às carreiras, tempo de serviço, pagamento, benefícios e outras questões específicas, cujas características para os funcionários da prefeitura são (SÃO PAULO, 1979):

Art. 2ㅇ - Para os efeitos deste Estatuto, funcionário público é a pessoa legalmente investida em cargo público. Art. 3 - Cargo público é aquele criado por lei, em número certo, com denominação própria, remunerado pelos cofres municipais, ao qual corresponde um conjunto de atribuições e responsabilidades cometidas a funcionário público. Art. 4으 - Classe é o agrupamento de cargos da mesma denominação e idêntica referência de vencimento. Art. 5o - Carreira é o conjunto de classes da mesma natureza de trabalho, escalonadas segundo a responsabilidade e a complexidade das atribuições. Art. 6ㅇ - Os cargos públicos são isolados ou de carreira. Art. 70 - Os cargos públicos são integrados em: I - Quadro Geral; II - Quadros Especiais, cujos cargos são agrupados por similitude das atividades neles compreendidas

A Lei Orgânica do Município de São Paulo (LOMSP), 01/90, rege a municipalidade e constitui a lei fundamental do município de São Paulo, conforme determinado na Constituição Federal (CF) (BRASIL, 1998) com o objetivo de organizar o exercício do poder e fortalecer as instituições democráticas e os direitos da pessoa humana. Destacam-se os artigos 212-218 do capítulo II da Saúde (SÃO PAULO, 1997):

\begin{abstract}
4ㅇ - É vedada a nomeação ou designação, para cargo ou função de chefia ou assessoramento na área de saúde, em qualquer nível, da pessoa que participe na direção, gerência ou administração de entidade ou instituição que mantenha contrato com o sistema único de saúde ou seja por ele creditada. Art. 217 - O sistema único de saúde do Município de São Paulo promoverá, na forma da lei, a Conferência Anual de Saúde e audiências públicas periódicas, como mecanismos de controle social de sua gestão. Art. 218 - Fica criado o Conselho Municipal de Saúde, órgão normativo e deliberativo, com estrutura colegiada, composto por representantes do Poder Público, trabalhadores da saúde e usuários que, dentre outras atribuições deverá promover os mecanismos necessários à implementação da política de saúde nas unidades prestadoras de assistência, na forma da lei.
\end{abstract}

O município também conta com um Código de Conduta Funcional dos Agentes Públicos e da Alta Administração Municipal, o Decreto no 56.130, de 26 de maio de 2015, apregoando normas de conduta funcional, de educação ética e de prevenção à corrupção, na conformidade das disposições deste decreto (SÃO PAULO, 2015).

As secretarias municipais de saúde-SMS, além de atuarem como gestoras do SUS municipal e repartições encarregam-se da formulação e implantação de políticas; de programas e projetos em prol da saúde populacional. No município de São Paulo, objeto desse estudo, a SMS se divide em Coordenadorias Regionais de Saúde-CRS, subdivididas por sua vez em supervisões, que fazem a gestão dos estabelecimentos de saúde. Todas essas instâncias compartilham em diferentes níveis a coordenação, articulação e organização do SUS municipal (SÃO PAULO, 2017).

As coordenadorias regionais de Saúde - CRS do município de São Paulo são responsáveis pela coordenação, articulação e organização de cada sistema local de saúde, ou seja, de cada divisão regional do município, são os distritos Regionais de Saúde: Centro, Oeste, Leste, Norte, Sudeste e Sul. além da compatibilização dos planos, programas e projetos dos Departamentos Regionais de Saúde (DRS) em função das políticas e diretrizes da SES/SP e dos recursos disponíveis. Com uma rede composta por: atenção básica, de especialidades, hospitalar, atendimento de urgência/emergência (SÃO PAULO, 2020).

A adoção de um índice de saúde distrital na cidade de São Paulo vai ao encontro da missão da Secretaria Municipal de Saúde de se comprometer com a transparência das suas ações, 
democratizando e divulgando para toda a sociedade os resultados alcançados pela atuação do SUS municipal.

O município também conta com a Coordenadoria de Gestão de Pessoas (COGEP) que inclui em suas ações de gestão de carreiras, assuntos de saúde, qualificação e bonificações para o servidos, é também responsável pelo planejamento, coordenação e implementação de políticas e ações de seleção, ingresso, gestão, integração, movimentação e desenvolvimento dos servidores, assim como diretrizes e normas para a gestão de pessoas, sua estrutura é composta por: Divisão de Desenvolvimento Profissional (DIDES), Divisão de Gestão de Carreiras (DICAR), Divisão de Gestão de Tempo de Serviço (DITEM) e Divisão de Gestão de Pagamento (DIPAG) (SÃO PAULO, 2020).

\section{PROCEDIMENTOS METODOLÓGICOS}

Trata-se de uma pesquisa descritiva, com abordagem qualitativa, realizada no município de São Paulo entre 2015 e 2017. A escolha dos entrevistados foi intencional e não aleatória, os dados foram coletados por meio de entrevistas semi-estruturadas que foram gravadas, transcritas e analisadas (BARDIN, 2004).

Com relação ao número de trabalhadores exercendo cargo de gestão a serem entrevistados, seis, Minayo (2004, p. 102) explica que em casos de metodologia qualitativa, o principal critério não é a quantidade de pessoas entrevistadas, e sim a reflexão que pode surgir da totalidade de suas múltiplas dimensões.

Nesse sentido, Bergue (2010a) salienta que a técnica de entrevista permite maior proximidade criando condições mais propícias à interação do pesquisador com o entrevistado, além de maiores esclarecimentos quanto aos requisitos necessários para a ocupação do cargo.

Diante da grande quantidade de conteúdo foi feito um trabalho complementar utilizando um aplicativo de nuvem de palavras, wordclouds ${ }^{1}$, o que proporcionou melhor visualização do impacto das palavras presentes na resposta às perguntas do questionário e serviu de apoio para análise das categorias.

Os entrevistados ( $A, B, C, D$ e E) das respectivas regionais, assinaram um Termo de Compromisso Livre e Esclarecido (TCLE), que deixava os participantes livres para recusar respostas a determinadas questões durante as entrevistas, bem como para retirar seu consentimento e terminar sua participação a qualquer momento, sem penalidades e sem prejuízo, garantindo o total anonimato dos dados colhidos.

O projeto foi aprovado no Comitê de Ética da Universidade Federal de São Paulo sob o número no 1475/2015 CEP/Unifesp e CAAE 51326215.7.3001.0086.

\section{RESULTADOS}

Todos os entrevistados das seis coordenadorias eram pós-graduados com formação para a função que estavam desenvolvendo no momento da entrevista, e já tinham exercido cargos anteriores de gestão. Apenas dois dos entrevistados não eram servidores públicos, ou seja, haviam sido contratados por cargo de livre provimento.

As entrevistas foram transcritas textualmente, o material foi lido e relido, os principais relatos foram destacados para facilitar o processo de análise.

\footnotetext{
${ }^{1}$ Aplicativo https://www.wordclouds.com
} 
Tabela 1 - Trechos das falas dos entrevistados

Entrevistados
“[...] difícil para a mentalidade do serviço público entender que existem outras formas
de gestão de pessoas, você pensa primeiro é formatado eu sou médica então é medica
minha área de atuação e normalmente eu sou um bom administrador porque eu sou
médico, e se eu for um médico bonzinho, seria um bom administrador, o que não tem
nada a ver, segundo que tem uma questão cultural pesada, de nível superior, existe
castas ainda, difícil achar que uma pessoa de nível médio pode ter uma atuação
diferente. Isso cria um mal-estar para a cultura da instituição e esse crescimento lateral
é uma coisa que as pessoas não estão habituadas [...]”.
"[...]então assim se você me perguntar que competência mais especifica eu acho que
tem a questão de sim do conhecimento de administração, de gestão de pessoas, de
gestão de conflitos, mas eu acho que é uma somatória muito grande tudo isso pra dizer
pra você especificamente ele tem que ter um nível universitário porque hoje também
tinha isso a gente não indicava se não tivesse um nível universitário acho que isso não
sei se agora caiu nessa gestão, mas eu sei que tem algumas pessoas também da área
administrativa que estão sendo indicadas como gerente já contrariando aquela coisa
inicial que a gente tinha que era só as pessoas formadas ai com ligações especificas na
área da saúde".

" [...] O problema é que os cursos relacionados a gestão pública geralmente, eles tem uma forte conotação de sistema único de saúde, coe faz o curso mais $60 \%$ são sobre o SUS, princípios, avanços, a pessoa vai com a expectativa de aprender gestão porem, a gestão mesmo é de 10 a $20 \%$ do curso, 70 a $80 \%$ sistema único de saúde, uma roda de conversa, ideias, mas uma vez aquelas ferramentas de gestão fica uma coisa menor, o importante é falar do sistema único de saúde, só que não fala que para o SUS ser viável,

C Se você não tiver ferramentas de gestão e financeira, ele não vai. É o que está acontecendo hoje, o financiamento já é pequeno [...] entender um pouco de gestão de pessoas, e não somente a gestão legal trabalhista, mas essa relação como se lida com o funcionário, é o tempo todo verticalizado ou não, como se lidera uma equipe, como se motiva, enfim a pessoa tem que ter um pouco de conhecimento, sem isso fica difícil discutir, o conhecimento ele é importante uma base teórica [...]

[...] entender um pouco de gestão de pessoas, e não somente a gestão legal trabalhista, mas, essa relação como se lida com o funcionário, é o tempo todo verticalizado ou não,

D como se lidera uma equipe, como se motiva [...] todo mundo deveria que ter uma boa gestão pública, uma gestão de planejamento, isso é fundamental.

"Na minha gestão eu analiso de duas formas: a competência individual da pessoa; a habilidade que essa pessoa tem para lidar com pessoas; porque na gestão de pessoas, você lida com gente, é a sua matéria prima é o seu cliente [...] “Os mesmos critérios, eu avaliava, eu gosto muito de trabalhar com os profissionais que já atuou na área, não teve na minha gestão um profissional que eu trouxe de outro lugar [...]eu acho que a gestão de pessoas é o mais importante [..]".

Os trechos das entrevistas foram extraídos para exemplificar os principais resultados da pesquisa, no que se refere à gestão de pessoas, na nuvem de palavras. 


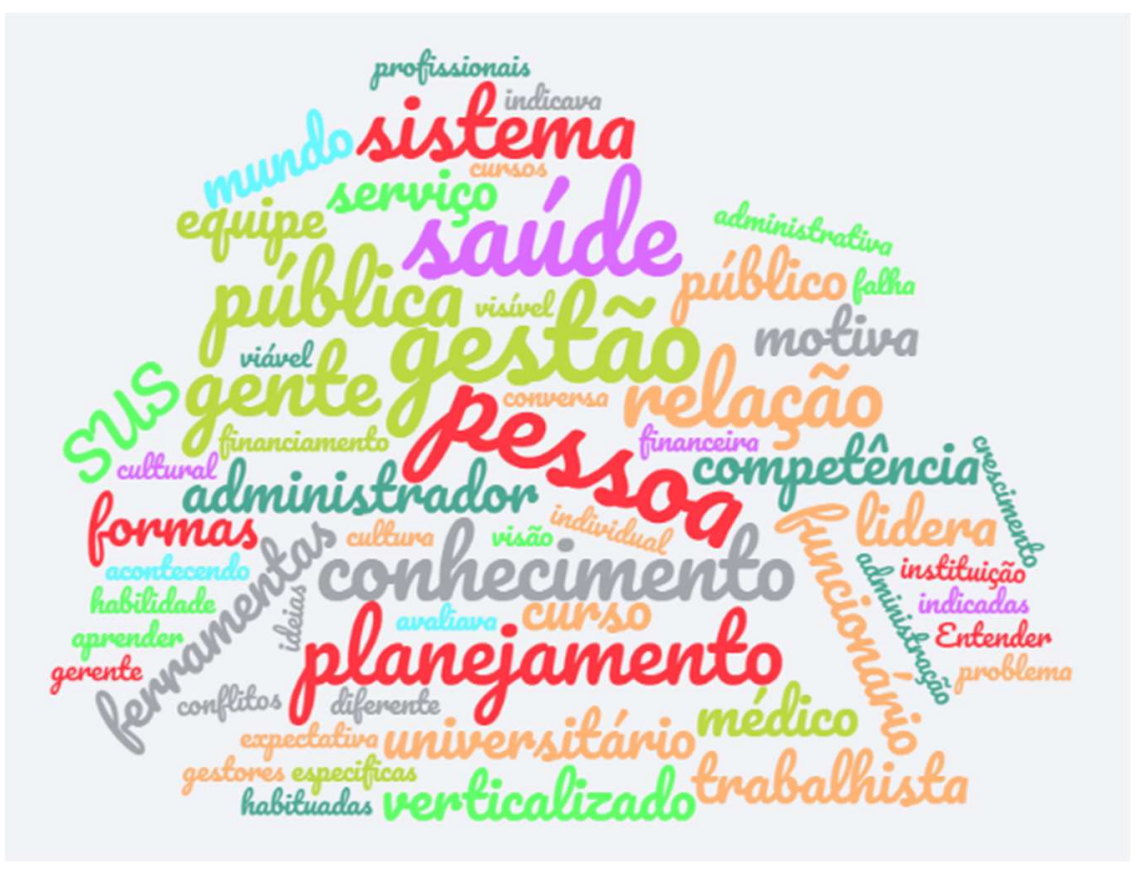

Figura 1: Representação gráfica das respostas dos entrevistados.

As palavras que ficaram em maior evidência foram: gestão, pessoa, saúde, conhecimento, gente e planejamento.

\section{DISCUSSÃO}

Os gestores que participaram da pesquisa foram consonantes no que se refere à gestão de pessoas no setor público. A nuvem de palavras indicou uma associação direta com as palavras: planejamento, gente, conhecimento, saúde, pessoas e gestão. Assim, pretende-se conduzir a discussão, de forma a buscar contemplar essas temáticas.

Mediante critérios pré-estabelecidos, destaca-se a importância da publicidade do edital, exigência legal interposta pelo art. 37 CF/88 como princípio da administração pública, que deveria estar associada aos princípios de impessoalidade, moralidade, legalidade e publicidade (BRASIL, 1998).

Para Bergue (2010b) nos editais devem ser especificados os temas cujo conhecimento será exigido na prova. Constituem-se como instrumento de seleção dos candidatos, incluindo também prova de títulos, que é considerada fundamental "a fim de que o processo seletivo cumpra seu objetivo, a adoção de instrumentos de avaliação adequados segundo a natureza e as particularidades dos cargos para os quais se pretende selecionar pessoal" (BERGUE, 2010a, p.538).

É fundamental que o processo seletivo ocorra dentro das normas legais objetivando evitar uma possível nulidade. Melo (2006) afirma ser indispensável determinar a exata quantificação de vagas a serem preenchidas, além do cargo ter sido criado por lei e que esteja vago para o concurso.

Aqui sobressai o princípio de igualdade de acesso aos cargos e empregos públicos, que deve repudiar as práticas de nepotismo e protecionismo. Conforme relatam Nogueira, Baraldi e Rodrigues (2004, p. 18) a aferição pública para admissão ao serviço público a) pode evitar o favorecimento, o apadrinhamento e diferentes formas de clientelismo; b) deve obedecer ao princípio do mérito, 
promovendo o acesso virtual a todos os cidadãos que tenham as competências requeridas; c) deve assegurar a transparência e a publicidade do processo de admissão.

Exprimindo-se sobre essas questões, o Conselho Nacional dos Secretários Estaduais de Saúde- CONASS (BRASIL, 2003) reconhece que o desenvolvimento de políticas de gestão de pessoas é um grande desafio para o SUS. Dessa forma diversos programas, portarias e políticas fazem parte desse processo tais como: HumanizaSUS, visando a gestão do cuidado e dos processos de trabalho e DesprecarizaSUS, que aborda o planejamento e desprecarização dos vínculos de trabalho no sistema público de saúde brasileiro (BRASIL, 2013; BRASIL, 2003).

A precarização dos vínculos de trabalho, bem como os problemas relacionados à gestão, é apontada por Lacaz et al. (2014) destacando-se a importância de os profissionais possuírem maior controle sobre o processo de trabalho. Segundo Merhy \& Feuerwerker (2009, p.11-13) os trabalhadores do SUS dependem da construção de processos relacionais ao trabalho e ao cuidado. Cabe observar ainda que, em um país como o Brasil, de porte continental, a gestão das organizações de saúde precisa saber lidar com as especificidades e a realidade de cada região.

Diante de tantas transformações, Ferreira e Araujo (2014) ressaltam que o SUS obteve significativas conquistas. No entanto, está, ao mesmo tempo, às voltas com limitações e impasses, pois ainda depende de uma conjunção de forças políticas que encarem a decisão de financiar adequadamente a saúde pública brasileira. Os autores consideram a dimensão do financiamento do SUS condição necessária, mas não suficiente, e sugerem a ampliação da gestão participativa e fomento das críticas, ativas e solidárias.

Embora nenhum dos entrevistados tenha usado os termos: eficiente, eficiência ou eficácia, segundo Paula (2005), as mudanças na gestão municipal do SUS, nos últimos anos, privilegiam a produtividade em detrimento da própria qualidade da atenção.

As secretarias municipais e estaduais de saúde, juntamente com órgãos e parceiros, têm um papel fundamental nas ações e mecanismos de operacionalização do funcionamento do SUS. A normatização do sistema de descentralização municipal possibilitou a contratação e gerência de trabalhadores, gerando maior necessidade de políticas de gestão do trabalho.

Pierantoni (2000, p. 52) frisa que "As formas verticalizadas da gerência são substituídas por gerência horizontal, com uma diminuição das funções de chefia e a introdução de sistemas mais participativos e abertos de decisão".

Várias iniciativas foram implementadas para o aprimoramento do SUS, e o Ministério da Saúde (BRASIL, 2002, p.41) destaca que novas competências são requeridas dos trabalhadores e gestores, demandando "novas formas de recrutar, selecionar, treinar e manter os profissionais em suas respectivas atividades, impondo a criação de instrumentos gerenciais essenciais a essa nova abordagem de gestão de recursos humanos", em busca de crescente especialização dos trabalhadores, com incidência direta na incorporação e na remuneração da força de trabalho.

Para os entrevistados as políticas de pessoal não são duradouras, e dessa forma, quando acontecem as mudanças de governo, normalmente a cada quatro anos, há uma desestabilização da equipe e, por conseguinte, do trabalho. Podem se tornar um divisor de águas, impactando diretamente na continuidade dos processos de gestão.

Dessa forma, a gestão do trabalho deve visar um modelo de gestão participativa, o que envolve reconhecer a grandeza dos processos e configuração da estrutura da área em que o trabalho se desenvolve, bem como as constantes mudanças que envolvem os trabalhadores e a sociedade (LACAZ, et al. 2014; NEUMANN, 2018). 


\section{CONSIDERAÇÕES FINAIS}

É fundamental que a Administração Pública utilize ferramentas que fomentem a transparência, divulgando informações de interesse público, já que a transparência pode estimular a participação da sociedade no processo de elaboração de políticas públicas.

Nota-se que os municípios, de uma forma geral, estão absorvendo cada vez mais responsabilidade pela atuação governamental em muitos aspectos, para regulamentar, intervir e atuar, à medida que adquirem autonomia estrutural e financeira, como é o caso da área da saúde.

O SUS tem uma importância fundamental como empregador, tanto com relação à força de trabalho quanto à grande diversidade de trabalhadores envolvidos na prestação de serviços de saúde, assim, a gestão de pessoas no serviço público acaba sendo vista como uma problemática que engloba outros fatores, tais como: saúde do trabalhador, qualidade de vida no trabalho. Temática essa que não pode ser contemplada aqui, mas espera-se desenvolvimento de intervenções que visem a melhora nos processos de trabalho.

Analisar a percepção dos gestores que contemplam regiões é um caminho promissor para a solução dos problemas emergentes e, para fortalecer a defesa dos interesses dos trabalhadores da área da saúde, demanda reflexões sobre as formas de ocupação de cargos públicos.

\section{AGRADECIMENTOS}

Agradecemos à CAPES-Coordenação de Aperfeiçoamento de Pessoal de Nível Superior, pela bolsa auxílio que permitiu tempo de dedicação ao projeto de pesquisa e ao doutorado.

\section{REFERÊNCIAS}

BARDIN, L. Análise de conteúdo. 3. ed. Lisboa: Edições 70, 2004.

BERGUE, S. T. Gestão de Pessoas em Organizações Públicas. Caxias do Sul, RS. Educs. 2010a.

BERGUE, S. T. Gestão de pessoas: bases teóricas e experiências no setor público. Organizado por Marizaura Reis de Souza Camões, Maria Júlia Pantoja e Sandro Trescastro Bergue. - Brasília: ENAP, 2010b.

BRASIL. Ministério da Saúde. Política de Recursos Humanos para o SUS: balanço e perspectivas, Brasília, DF, 2002.

BRASIL. Conselho Nacional de Secretários de Saúde, CONASS. Para entender a gestão do SUS. Brasília, DF, 2003.

BRASIL. Ministério da Saúde. Política Nacional de Humanização - PNH - HumanizaSUS, Portaria GM/MS no 2406, 19.12.2003, Brasília-DF, 2013.

BRASIL. Constituição da República Federativa do Brasil - CF. Brasília, DF, Senado Federal: Centro Gráfico, 292 p. 1988. 
BRESSER-PEREIRA, L. C. A reforma administrativa do Sistema de Saúde. Cadernos n.13 da Reforma do Estado. Ministério da Administração e Reforma do Estado. Brasília, DF, 1998.

COHN A. A reforma sanitária brasileira após 20 do SUS: reflexões. Cad. De saúde pública, v. 25, n. 7, p. 1614-1619, 2009.

FERREIRA J. L. N.; ARAÚJO, J. N. G. Gestão e subjetividade no SUS: o enfrentamento de impasses em tempos neoliberais. Psicologia \& Sociedade, v. 26, n. 3, 2014.

LACAZ, F.A.C. et al. Gestão do Trabalho em Saúde em dois Sistemas Municipais do Estado de São Paulo: implicações operacionais e psicossociais para uma política de pessoal. Relatório Técnico. São Paulo: Fapesp, 2014.

LACAZ, F.A.C.; JUNQUEIRA, V. Gestão do Trabalho, Estado de Bem-Estar Social, Neoliberalismo: Estado "Mínimo" e a Saúde dos Trabalhadores do SUS. In: LACAZ, F.A.C; GOULART P.M. e JUNQUEIRA V.(orgs). Trabalhar no SUS, p. 29-64, 2017.

MACHADO, M. H.; FILHO, W. A. Gestão do trabalho em saúde: a evolução do conceito e suas implicações para o setor saúde no Brasil IN. Saúde, Trabalho e Cidadania em Mato Grosso. EduFMT, Cuiabá-MT, pp. 53- 66, 2014.

MELO, F.J.G. Admissão de Pessoal no Serviço Público. Procedimentos, restrições e Controles. (De acordo com a Lei de Responsabilidade Fiscal), Belo Horizonte: Editora Fórum, 2006.

MERHY, E. E.; FEUERWERKER, L. C. M. Novo olhar sobre as tecnologias de saúde: uma necessidade contemporânea. Leituras de novas tecnologias e saúde. São Cristóvão: Editora UFS, v. 1, 2009.

MINAYO, M.C.S. O desafio do conhecimento. Pesquisa Qualitativa em saúde. 8a edição, São Paulo: Editora Hucitec, 2004.

NEUMANN, Alessandra Paula Ferreira Moreira. Administração pública em saúde e a ocupação de cargos de gestão: análise de dois municípios de grande porte do Estado de São Paulo e uma província canadense. 263 p. [Tese de doutorado] Universidade Federal de São Paulo: São Paulo, 2018.

NOGUEIRA, R. P.; BARALDI, S.; RODRIGUES, V. A. Limites críticos das noções de precariedade e desprecarização do trabalho na administração pública. Observatório de recursos humanos no Brasil: estudos e análise. Brasília: Ministério da Saúde, NESP/CEAM/UnB, 2004.

PAULA, A. P. P. Administração pública brasileira entre o gerencialismo e a gestão social. RAE-Revista de Administração de Empresas, v. 45, n. 1, p. 36-49, 2005.

PIERANTONI, C.; Reformas da saúde e recursos humanos: Novos desafios x velhos problemas. 228 p. [Tese Doutorado] - Universidade do Estado do Rio de Janeiro, Instituto de Medicina Social, Rio de Janeiro, 2000. 
SÃO PAULO, Coordenadoria de Gestão de Pessoas - COGEP. 2019. Disponível em: <https://educacao.sme.prefeitura.sp.gov.br/cogep/>. Acesso em: 11 ago 2020.

SÃO PAULO. Secretaria municipal de saúde. Distritos de saúde - DS, 2017. Disponível em: <http://www.prefeitura.sp.gov.br/cidade/secretarias/saude/organizacao/index.php? p=6541> Acesso em: 11 ago 2020.

SÃO PAULO. Decreto no 56.130 de 26 de maio de 2015, Código de Conduta Funcional dos Agentes Públicos e da Alta Administração Municipal. São Paulo, 2015. Disponível em: <http://www.prefeitura.sp.gov.br/cidade/secretarias/upload/chamadas/d_56130_1432738091.pdf > Acesso em: 05 set 2020.

SÃO PAULO. Secretaria municipal de saúde. Servidor público estadual, SES/SP, Cartilha do observatório de Recursos Humanos, n. 9, edição especial, outubro, 2013. Disponível em:<http://www.saude.sp.gov.br/resources/crh/ggp/cartilhas/cartilha9servidorpublico. pdf> Acesso em: 11 ago 2020.

SÃO PAULO. Secretaria municipal de saúde. Coordenadoria de Recursos Humanos-CRH. São Paulo. 2012. Disponível em <http://saude.sp.gov.br/coordenadoria-de-recursoshumanos/crh/apresentacao/ apresentacao> Acesso em: 12 ago 2020.

SÃO PAULO. Lei orgânica do município 01/90, versão integral e consolidada. São Paulo, 1997. Disponível em: <http://www.prefeitura.sp.gov.br/cidade/secretarias/upload/chamadas/ leiorganica_1328215630.pdf $\gg$ Acesso em: 28 set 2020.

. Lei no 8.989, de 29 de outubro de 1979. O Estatuto dos Servidores Públicos do Município de São Paulo. Disponível em:

<http://www.prefeitura.sp.gov.br/cidade/secretarias/gestao/portal_do_servidor/estatuto_do_serv idor/index.php?p=10687> Acesso em: 28 set 2020.

SCHRAIBER, L. B. et al. Planejamento, gestão e avaliação em saúde: identificando problemas. Ciência \& Saúde Coletiva, v. 4, p. 221-242, 1999. 